\title{
Inverse-problem-based algorithm for sparse reconstruction of Terahertz off-axis holograms
}

\author{
Murielle Kirkove, Yuchen Zhao, Marc P. Georges \\ Centre Spatial de Liège, STAR Research Unit, Université de Liège, Liege Science Park, B-4031 Angleur, Belgium \\ m.kirkove@uliege.be
}

\begin{abstract}
We propose a new inverse-problem-based reconstruction technique to off-axis $\mathrm{THz}$ digital holography. The proposed method takes into account the non-uniformity of the reference wavefront and reconstructs the object field as well as the amplitude of the reference field. (C) 2021 The Author(s)
\end{abstract}

\section{Introduction}

Terahertz (THz) imaging techniques have been growing for the last decades. Various imaging techniques have been successfully migrated in the THz band [1]. Off-axis digital holography (DH) was one of the first techniques in the coherent lensless imaging family implemented in the THz band [2] since it is a mature configuration widely used in the visible wavelength. However, a few particular problems we encountered in $\mathrm{THz}$ off-axis DH differ from the configuration under the visible light. The diffraction effect is significant in $\mathrm{THz}$ range. The recording distance should be minimized to several centimeters to get a reasonable lateral resolution. Such a limited configuration makes the injection of reference wave more difficult. Any obstruction of the injected reference beam will cause excessive unwanted diffraction fringes which degrade the reference wave uniformity. Secondly, the penetration ability of $\mathrm{THz}$ radiation makes $\mathrm{THz} \mathrm{DH}$ advantageous for observing concealed objects. The border effect caused by the light field truncation when observing objects in $\mathrm{THz}$ severely degrades the imaging quality of the reconstruction. As importantly, the performance of $\mathrm{THz}$ cameras for hologram recording is not comparable to the visible cameras. At the far-infrared (FIR) band, the thermal focal plane arrays are employed, whereas suitable large pixel arrays are still not readily available for sub- $\mathrm{THz}$ hologram recording. The above-mentioned problems inevitably deteriorate the reconstruction quality. Earlier, we have attempted solving the DH reconstruction problem [3] via a easy-to-implement inverse problem (IP) approach proposed in [4]. The encouraging results suggested that the IP approach not only suppresses the unwanted border effect; it is also more tolerant to the noise, sub-sampling and the harsh recording conditions compared to the standard direct reconstruction. In this contribution, we propose a new IP approach for DH reconstruction. The reconstruction problem formulation is sparsified by the wavelet representation.

\section{Algorithm description}

The aim of IP DH reconstruction is to estimate the complex object wavefront $\boldsymbol{\psi}$ from the obtained holograms $y$ (the measurements). The forward model of the off-axis DH can be formulated as

$$
\boldsymbol{y}=\left|\boldsymbol{A}_{d} \boldsymbol{\psi}+\boldsymbol{\alpha} \boldsymbol{r}\right|^{2}+\boldsymbol{n},
$$

where $\boldsymbol{A}_{d}$ is the propagator of a distance of $d$ derived from the angular spectrum method and $\boldsymbol{n}$ represents the additive noise. An extra parameter $\boldsymbol{\alpha}$ is a scalar map to be estimated, representing the relative intensity of the unit reference beam $\boldsymbol{r}$, aiming to cope with the non-uniformity issue. Accordingly, the data-fidelity term $D$ to be minimized is

$$
(\tilde{\boldsymbol{\psi}}, \widetilde{\boldsymbol{\alpha}})=\underset{\boldsymbol{\psi}, \boldsymbol{\alpha}}{\operatorname{argmin}} D(\boldsymbol{\psi}, \boldsymbol{\alpha}), D(\boldsymbol{\psi}, \boldsymbol{\alpha})=\frac{1}{4}\left\|\boldsymbol{y}-\left|\boldsymbol{A}_{d} \boldsymbol{\psi}+\boldsymbol{\alpha} \boldsymbol{r}\right|^{2}\right\|_{2}^{2} .
$$

The problem is ill-posed. Regularization is mandatory for selecting meaningful solutions. We regularize both variables $\psi$ and $\alpha$ in the wavelet domain by minimizing the $l_{1}$-norm of their wavelet coefficients for promoting sparse solutions [5,6]. We express the variables $\boldsymbol{\psi}$ and $\boldsymbol{\alpha}$ in the wavelet domain:

$$
\boldsymbol{\psi}=\boldsymbol{W}_{\psi}^{-1} \boldsymbol{c}_{\psi}, \boldsymbol{\alpha}=\boldsymbol{W}_{\alpha}^{-1} \boldsymbol{c}_{\alpha},
$$

where $\boldsymbol{W}_{\psi}\left(\boldsymbol{W}_{\alpha}\right)$ is the matrix of fast discrete wavelet transform (DWT) for $\boldsymbol{\psi}(\boldsymbol{\alpha})$ providing the vector of the wavelet coefficients $c_{\psi}\left(\boldsymbol{c}_{\alpha}\right)$. Taking into consideration of Eq. (2) as well as the regularization terms, the reconstruction problem becomes :

$$
\left(\tilde{c_{\psi}}, \tilde{c_{\alpha}}\right)=\underset{c_{\psi}, c_{\alpha}}{\operatorname{argmin}} D\left(c_{\psi}, c_{\alpha}\right)+\lambda_{\psi}\left\|\tilde{c_{\psi}}\right\|_{1}+\lambda_{\alpha}\left\|c_{\alpha}\right\|_{1}
$$


where $\lambda_{\psi}$ and $\lambda_{\alpha}$ control the strengths of the regularization terms. The biorthogonal Cohen-Daubechies-Feauveau (CDF) 9/7 wavelet was used for performing the DWT, which is broadly used in the JPEG 2000 compression standard for lossy compression. It was recommended in [5] for compressive DH reconstruction for its sparse representations and its robustness when performing diverse DH reconstruction problems. The sum of the three terms in equation (4) are optimized with a dedicated algorithm using an alternating direction method of multipliers (ADMM) based framework.

\section{Results}

Fig. 1 shows the results of the proposed method applied on synthetic and experimental data. The synthetic data are obtained by simulating a highly transparent phase object and modeling the non-uniformity of the amplitude of the reference field by a Gaussian function. The reconstructed amplitude and phase are shown in Fig.1(a-b) for the synthetic hologram and in Fig.1(c-d) for the hologram recorded at $2.52 \mathrm{THz}$.

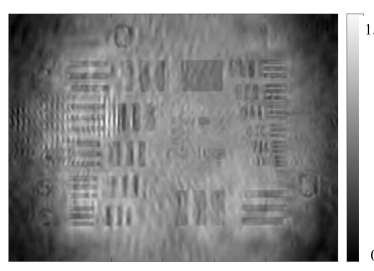

(a)

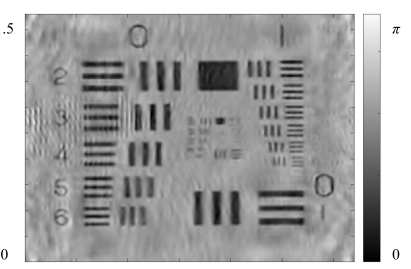

(b)

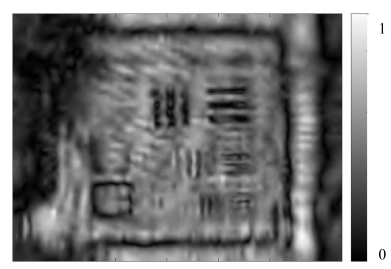

(c)

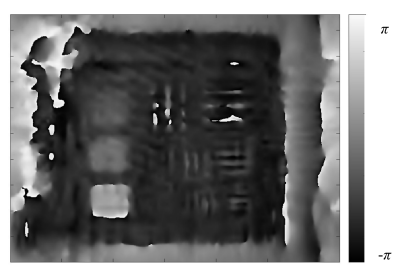

(d)

Fig. 1. Proposed method applied on synthetic (a-b) and real (c-d) data

\section{Conclusions and future works}

The proposed algorithm shows the benefits of IP approach during reconstruction. The convergence and the parameter tuning are more reliable compared to [4]. The ADMM framework takes care of the data-term and the regularization terms for both the object wavefront reconstruction and the reference amplitude restoration. The retrieved reference amplitude distribution is credible since its form resembles the observation. It is not quantitatively correct yet. This is understandable, current data-term contains only one measurement: the hologram, where the object wavefront and the reference wavefront contribute equally to the hologram. We cannot correctly separate them based on the sole measurement. However, if additional measurement of object diffraction pattern is added to the data-term, a good separation can be expected. The wavelet decomposition applied in the proposed algorithm can be particularly useful for image reconstruction since adaptive regularization can be applied with respect to the awaited resolution. The development of the proposed algorithm is inspired by the difficulties that we encountered in $\mathrm{THz} \mathrm{DH}$, it is independent to the recording wavelengths. The potential of proposed algorithm will be studied in future works.

\section{Funding}

This research is funded by project TERA4ALL in the frame of 2014-2020 ERDF/Wallonia region "En Mieux" program.

\section{References}

1. D. M. Mittleman, “Twenty years of terahertz imaging," Opt. Express 26, 9417-9431 (2018).

2. L. Valzania, Y. Zhao, L. Rong, D. Wang, M. Georges, E. Hack, and P. Zolliker, “Thz coherent lensless imaging,” Appl. Opt. 58, G256-G275 (2019).

3. Y. Zhao, M. Kirkove, and M. P. Georges, "Inverse-problem based algorithm for thz off-axis digital holography reconstruction," in Imaging and Applied Optics Congress, (Optical Society of America, 2020), p. HF4G.6.

4. A. Bourquard, N. Pavillon, E. Bostan, C. Depeursinge, and M. Unser, "A practical inverse-problem approach to digital holographic reconstruction," Opt. Express 21, 3417-3433 (2013).

5. S. Bettens, H. Yan, D. Blinder, H. Ottevaere, C. Schretter, and P. Schelkens, "Studies on the sparsifying operator in compressive digital holography," Opt. Express 25, 18656-18676 (2017).

6. C. Schretter, D. Blinder, S. Bettens, H. Ottevaere, and P. Schelkens, "Regularized non-convex image reconstruction in digital holographic microscopy," Opt. Express 25, 16491-16508 (2017). 